Cahiers Société

\title{
Réalité, strates et chaos : enjeux historiographiques de l'oeuvre de Cornelius Castoriadis
}

\section{Nicolas Piqué}

Numéro 1, mai 2019

Castoriadis et les sciences sociales

URI : https://id.erudit.org/iderudit/1068424ar

DOI : https://doi.org/10.7202/1068424ar

Aller au sommaire du numéro

Éditeur(s)

Collectif Société

ISSN

2562-5373 (imprimé)

2562-5381 (numérique)

Découvrir la revue

Citer cet article

Piqué, N. (2019). Réalité, strates et chaos : enjeux historiographiques de l'oeuvre de Cornelius Castoriadis. Cahiers Société, (1), 153-172.

https://doi.org/10.7202/1068424ar 


\title{
Réalité, strates et chaos : enjeux historiographiques de l'œuvre de Cornelius Castoriadis
}

\author{
Nicolas PIQUÉ \\ Université de Grenoble
}

Un auteur ne se résume pas seulement à la somme de ses propositions et de ses thèses, dont il est alors possible de rendre compte de manière interne et analytique. Une œuvre comporte également ce que l'on pourrait appeler un pouvoir d'orientation. Elle permet de créer des perspectives généalogiques ou rétrospectives (comprises, on y reviendra, par Castoriadis sous les auspices de la notion de "pensée héritée »), elle rend aussi possible de repérer des polarités dans des champs au sein desquels elle permet alors de se situer. Ce sont ces pouvoirs généalogique et heuristique de l'œuvre de Cornelius Castoriadis qui seront sollicités dans les remarques qui vont suivre. Je m'y suis moins attaché à élucider ses positions que je m'en suis servi pour repérer des singularités qu'elles permettaient de faire apparaître, comme en résonance.

Il s'est donc agi de sonder la façon dont ses concepts et ses positions théoriques peuvent permettre, à partir de leur singularité, de repérer des programmes de recherche tout aussi particuliers; de dépasser le cadre doxographique pour circonscrire des usages, non pas en repérant des influences explicites ou revendiquées - ce serait un autre travail -, mais discriminer ceux des programmes de recherche qui entrent en résonance en quelque sorte avec ce que Castoriadis contribue à penser de façon plus théorique.

On se concentrera ici sur le champ historique en prenant les positions de Castoriadis comme guide de lecture pour repérer comment, dans la pratique même de l'écriture de l'histoire, on peut être attentif à l'historicité indépassable de la réalité social-historique. Plus précisément, c'est donc à partir des analyses que propose Castoriadis des notions de chaos et de strates irrégulières qu'ont été abordées, dans la suite de cet article, les références à l'histoire de la philosophie tout autant que les paradigmes historiques plus contemporains. D'abord parce qu'elles occupent, dans le corpus castoriadien, une place théorique fondamentale ; mais aussi parce que, en plus de leur importance conceptuelle interne, elles constituent également les conditions d'un paradigme historiographique tout à fait singulier. 
Réalité, strates et chaos : enjeux historiographiques de l'œuvre de Castoriadis | Nicolas PIQUÉ

\section{Thèses : chaos et strates irrégulières}

Il ne saurait être question, ici, d'analyser de manière systématique les propositions de Castoriadis, d'en étudier la genèse dans ses textes, d'en éprouver la cohérence ou d'en tester les limites. Il s'agira, plus modestement, de repérer et circonscrire les éléments théoriques concernant l'histoire dont les attendus, interprétatif et généalogique puis heuristique et contemporain, pourront ensuite être testés, comme matrice de lecture.

Commençons par présenter la radicalité et la singularité des positions de Castoriadis relatives à l'histoire : elles permettent d'échapper à une conception éternitiste de l'histoire. On reviendra plus loin sur ce barbarisme, qui me parait toutefois nécessaire : la pensée héritée a longtemps analysé l'histoire à partir ou en vue d'un possible et nécessaire dépassement de l'histoire. Cette dernière n'a longtemps été étudiée qu'en fonction de lois tendant à faire apparaître des déterminations anhistoriques et surplombantes : étudier l'histoire pour échapper à l'historicité en quelque sorte. Or les positions de Castoriadis conduisent, à l'inverse, à la critique radicale d'une telle entreprise. La stratification irrégulière de la réalité social-historique et l'ontologie chaotique interdisent tout espoir de dialectisation, tout espoir d'une position de surplomb stable, à même de dessiner ou de dresser la carte des possibles.

La notion de chaos est souvent mise de l'avant par Castoriadis lui-même dans ses écrits. On peut penser aux diverses formulations, assez solennelles, de "thèses », c'est le terme alors employé1, qui lui permettent de caractériser l'être comme « Chaos », "Abîme » ou "Sans-Fond" ». Cette position ontologique a pour enjeu fondamental de remettre en cause le paradigme essentiel de la déterminité, qui caractérise, limite et réduit la philosophie depuis Platon. Le caractère chaotique de l'être empêche toute réduction déterministe au profit d'une approche nouvelle, dont Castoriadis pense et présente les nécessaires nouveaux concepts. Parmi ceux-ci, en rappelant encore le caractère schématique de cette présentation, on se concentrera sur la notion de stratification irrégulière. C'est dans « La logique des magmas et la question de l'autonomie » et dans « Portée ontologique de l'histoire des sciences3 » que le caractère irrégulier est souligné alors même que la notion de stratification apparaît plus tôt, en particulier dans «Science moderne et interrogation philosophique4 ». Cette caractérisation est décisive pour rendre compte et du caractère chaotique de l'être et de l'impossible clôture de la réalité, autres notions décisives ; c'est aussi à

\footnotetext{
${ }^{1}$ Cornelius Castoriadis, «La logique des magmas et la question de l'autonomie », dans Les carrefours du labyrinthe, II. Domaines de l'homme, Paris, Seuil, 1978, p. 385, Castoriadis parle ailleurs d' «affirmations tout à fait dogmatiques » («L'imaginaire: la création dans le domaine socialhistorique », dans Les carrefours du labyrinthe, II. Domaines de l'homme, op. cit., p. 219).

${ }^{2}$ Idem.

3 Ce sont les deux derniers articles publiés dans Les carrefours du labyrinthe, II. Domaines de l'homme, op. cit.

4 En 1970-1971 donc ; texte repris dans Les carrefours du labyrinthe, I, Paris, Seuil, 1978.
} 
cette irrégularité, à cette complexité que sont sensibles les historiens que nous allons lire.

Si l'historicité ne peut être relevée par une dialectique dépassant l'histoire, c'est en raison de cette stratification dont l'irrégularité (non pas accidentelle ou fortuite, mais fondamentale) interdit d'envisager une position ou posture théorique à partir de laquelle l'ordonner, la stabiliser et la réduire. Cette irrégularité relève quant à elle de l'impossibilité pour l'être d'être systématisé. Historicité fondamentale, irrégularité et chaos sont donc liés.

L'indétermination foncière de la réalité5 se trouve ainsi caractérisée, rendant compte aussi de la densité. Chaque strate peut en effet être accessible à un mode de causalité, mais leur ensemble échappe essentiellement, fondamentalement à une prise globale, pérenne et consistante. Apparaît alors une dualité décisive pour le travail de Castoriadis. Sa singularité philosophique (plus encore rapportée à l'histoire) réside en effet dans la double reconnaissance et de l'historicité essentielle de l'être et des moments de stabilité régionale ou épocale. On reconnaît là la double tâche de pensée souvent tracée par Castoriadis, par exemple quand il insiste sur le nécessaire double questionnement : «Deux questions fondamentales surgissent dans le domaine socialhistorique. Premièrement, qu'est-ce qui tient une société ensemble ? [...] Deuxièmement, qu'est-ce qui fait surgir des formes de sociétés autres et nouvelles ${ }^{6}$ ? »

Ce programme intéresse tout particulièrement l'historien, confronté à la fois à la stabilité relative d'une époque, d'une société, et à la façon dont jamais aucune institution de sens n'est totalement pérenne, quoi qu'elle en dise ou prétende. Une telle historicité résulte de la dimension chaotique de l'être ; elle est également le résultat du caractère irrégulier de la stratification de la réalité. La notion de densité, telle que définie par Castoriadis, rend également compte de cette double dimension, en permettant de dépasser les oppositions héritées (universel/relatif, ordre/changement, parmi d'autres), de manière toutefois non dialectique.

Ces précisions ne sont guère que des rappels pour tout lecteur de Castoriadis. Elles n'ont d'autre but que de clarifier les positions à partir desquelles mettre en œuvre leur portée interprétative et heuristique. Interprétative dans leur capacité à revenir sur et circonscrire ce que Castoriadis appelle la pensée héritée : il s'agira, dans un premier moment, de repérer comment ces schèmes de pensée rompent avec les paradigmes préexistants (chez Bossuet, Kant, Herder, E. Troeltsch ou C. Schmitt pour la notion de chaos), de localiser aussi des usages précurseurs (chez G. Cuvier et F. Nietzsche pour la notion de strate). Ils proposent une autre systématicité, permettant de discriminer, de saisir la puissance d'impensé et de déni à l'œuvre dans

5 On trouve dès L'institution imaginaire de la société une formulation de ce type, Paris, Seuil, 1999, p. 513.

${ }^{6}$ Cornelius Castoriadis, «L'imaginaire : la création dans le domaine social-historique », dans Les carrefours du labyrinthe, II, op. cit., p. 222. 
l'histoire de la philosophie. Heuristique, dans un deuxième moment, consacré davantage à la lecture d'historiens7. On y tentera une sorte de cartographie de quelques paradigmes historiographiques contemporains, analysant successivement les positions et pratiques de C. Ginzburg, d'E. P. Thompson et des promoteurs de l'histoire connectée. Ce sera alors l'occasion de discuter de la dimension possiblement architectonique des propositions de Castoriadis.

C'est à partir de ces positions que l'on pourra repérer dans les travaux de ces historiens le lien entre strate, chaos et dimension irrémédiablement dynamique de la réalité historique ; c'est aussi à partir de ces positions qu'il sera possible de dépasser, du point de vue historique, la pensée héritée. De telle sorte que l'analyse de la réalité par le biais de ce schème de la strate permet d'échapper au paradigme aristotélicien, pour lequel le repos constitue la fin du mouvement et le stable anhistorique, la fin de l'historicité.

La notion de " pensée héritée 8 », à laquelle Castoriadis se réfère de manière régulière et structurante, est d'un usage délicat, toujours susceptible de schématiser et réduire un ensemble de références irréductiblement plus divers que ne pourrait le laisser penser cette notion. Je m'y risquerai toutefois ici pour caractériser la pensée héritée de l'histoire par l'hypothèse éternitiste qui vient d'être évoquée, par un paradigme anhistorique : l'analyse de l'histoire, au sens d'ensemble des événements passés, vise à faire apparaître du stable, à faire advenir de l'ordre au-delà du chaos événementiel. Le chaos y est pensé comme défaut d'ordre, menace morale ou scandale rationnel. Loin de toute visée exhaustive, les références qui vont suivre (philosophes scrutant l'histoire ou historiens la pratiquant) sont là pour exemplifier et caractériser la pensée héritée ; pour souligner aussi son inertie, au-delà même des limites chronologiques auxquelles on pense pouvoir la circonscrire.

\section{Chaos ou profondeur}

Pour nous en tenir à l'époque moderne, le Discours sur l'histoire universelle de Bossuet en propose une expression presque exemplaire quand il écrit que «par le temps

\footnotetext{
7 Castoriadis souligne les attendus historiques, voire historiographiques de son ontologie : « nous avons à comprendre que l'être est stratifié essentiellement - et cela, non pas une fois pour toutes, mais "diachroniquement" ». Cf. Cornelius Castoriadis, "Portée ontologique de l'histoire de la science », dans Les carrefours du labyrinthe, II, op. cit., p. 455.

8 Rappelons que par cette notion Castoriadis désigne l'ensemble des systèmes composant l'histoire de la philosophie, ensemble caractérisé par des propositions dont il se démarque fortement. Ayant trait principalement au principe de détermination, elles lui apparaissent incapables de saisir la dimension chaotique de l'être, caractéristique pour lui essentielle et fondamentale tant pour ses attendus épistémologiques que politiques. Voir le commentaire que propose Vincent Descombes de ce schème dans «Un renouveau philosophique », dans Autonomie et autotransformation de la société : la philosophie militante de Cornelius Castoriadis, dir. Giovanni Busino, Genève, Droz, 1989, p. 69-85.
} 
elle [l'étude historique de la suite de l'Église] vous mène à l'éternité9 ». Analyser l'histoire n'a de sens qu'à y faire apparaître un ordre anhistorique sans lequel la vie humaine ne saurait avoir de sens. Une même tension se retrouve chez Kant au début de L'Idée d'une histoire universelle d'un point de vue cosmopolitique, où l'enjeu de l'étude de l'histoire est présenté comme lieu de recherche de stabilité ; sans stabilité, c'est le chaos et le désespoir moral qui guettent, sous les apparences d'une « forme embrouillée et irrégulière » ou de la « situation chaotique où se trouvent les relations entre États ». Cette défiance morale du chaos se retrouve encore chez Herder, qui fait $\mathrm{du}$ « doute de la vertu » et du « scepticisme à l'égard de toute vertu» la conséquence $\mathrm{du}$ « $\operatorname{chaos}^{10}$ » que manifeste le défaut de prise en compte de plan de progression dans l'histoire. Le même dilemme agite Voltaire dans le « Poème sur le désastre de Lisbonne » : le spectacle du tremblement de terre comme plus généralement celui de l'histoire du monde permettent de conclure que « tout est en guerre ${ }^{11}$ » et que domine le « chaos de malheur », le « chaos fatal12 ».

À chaque fois, l'enjeu et le sens de l'analyse de l'histoire sont présentés dans le cadre d'une opposition au chaos ${ }^{13}$. La pensée historique héritée reste prise dans un horizon épistémologique nomothétique ou dans une approche morale ; le chaos y est pensé comme ce qui fait échec à la pensée, comme ce qui menace aussi la possibilité et le sens d'une morale.

Dans le cadre de la pensée héritée, l'histoire relève donc de la recherche d'un point d'Archimède à même de stabiliser l'histoire, à même d'en faire surgir le sens dans une perspective qui ne peut être qu'anhistorique. Deux dernières références vont souligner combien même les coups de semonce de Nietzsche14, dans La généalogie de la morale en particulier, n'auront pas suffi à épuiser ce paradigme.

La visée ordonnatrice que l'on a dégagée se retrouve encore chez Ernst Troeltsch, qui en fait l'un des enjeux de la crise de l'historisme : «Par son relativisme qui exprime et comprend tout [...] l'histoire a préparé la voie à cet ébranlement des valeurs ${ }^{15}$ ». La crise est radicale («L'État, le droit, la morale, la religion, l'art, tout est dissout $[\mathrm{sic}]$ dans le fleuve du devenir historique, et nous ne pouvons dorénavant les comprendre que comme des éléments inscrits dans des développements histori-

\footnotetext{
9 Jacques-Bénigne Bossuet, Discours sur l'histoire universelle, II, 31.

10 Johann Gottfried Herder, Une autre philosophie de l'histoire, Paris, Aubier, 1964, p. 187, 193.

${ }^{11}$ Voltaire, « Poème sur le désastre de Lisbonne », dans Mélanges, Paris, Gallimard, 1961, p. 307.

12 Ibid., p. 308.

13 Marx encore pense dans ce cadre, peut-être en partie seulement. Dans l'Introduction à la critique de l'économie politique (Paris, Éditions sociales, 1972), il caractérise comme « représentation chaotique du tout » (p. 165) la pensée des économistes classiques là où sa propre logique évolutionniste (dans ce texte en tout cas) permet d'établir et fonder une conception continuiste et ordonnée de l'histoire.

14 Voir le commentaire qu'en propose Michel Foucault dans « Nietzsche, la généalogie, l'histoire», dans Hommage à Jean Hyppolite, Paris, Presses Universitaires de France, 1971, p. 145-172.

15 «La crise de l'historisme », dans Religion et histoire, dir. Jean-Marc Tétaz, Genève, Labor et Fides, 1990, p. 206.
} 
ques $\left.^{16} \gg\right)$, le désarroi total. Face à cette rhapsodie, que convient-il de faire ? Comment accepter ce «polythéisme des valeurs ${ }^{17}$ », problématique tant il rend difficile l'orientation dans un monde ayant perdu tout « gouvernail18 »? Face à ce bouleversement, cet ébranlement, cette secousse (les termes abondent chez Troeltsch pour décrire cette crise des valeurs), face au constat que « [t]out est en lutte contre tout19 », une seule solution, il devient urgent de retrouver « la voie qui mène de l'historique relatif à des valeurs culturelles effectives ». " Il s'agit de construire à partir des études historiques le fondement d'un système culturel présent, qui assurera aussi le prochain avenir »; « il s'agit de dépasser l'histoire par l'histoire ${ }^{20} »$. Aussitôt entrevue ${ }^{21}$, la représentation proprement historique de la temporalité, caractérisée par l'acceptation de discontinuité et donc par la remise en cause des notions essentialisées, est immédiatement recouverte par une recherche synthétique dont le but est de retrouver une stabilité. Pour Troeltsch, il est humainement impossible de vivre dans un monde désorienté, c'est-à-dire dans un monde qui aurait perdu sa stabilité et son universalisme traditionnel ${ }^{22}$.

Dernière occurrence chez Carl Schmitt, plus tard encore, lorsqu'il présente la notion de katéchon: même si la notion de chaos n'apparaît pas explicitement chez lui, l'opposition chaos-ordre structure bien un ensemble de passages qui compose ce que l'on pourrait appeler la théorie du katéchon. Il s'agit d'une série de références au deuxième chapitre de la deuxième Épittre aux Thessaloniciens de Paul: dans le contexte de l'attente de la venue de Jésus, il convient de ne pas s'agiter (v. 2), le « mystère d'Iniquité est déjà à l'œuvre » (v. 7). Paul conclut : « Et maintenant vous savez ce qui le retient pour qu'il ne soit dévoilé qu'en son temps » (v. 6). À plusieurs reprises, Schmitt se réfère à cette assez mystérieuse force de rétention (en quoi consiste le katéchon), en soulignant l'enjeu consistant à s'opposer à l'accélération de l'histoire : «Je crois au katéchon, c'est pour moi la seule possibilité de comprendre l'histoire en chrétien et de lui trouver du sens ${ }^{23} \gg$. La pensée du katéchon seule permet alors d'échapper à « l'accroissement entropique du chaos du monde 24 ».

16 Ibid., p. 226.

17 Ibid., p. 220.

18 Ibid., p. 219.

19 Ibid., p. 220.

20 Ernst Troeltsch, cité par Jean-Marc Tétaz, « Religion et conscience historique », dans ibid., p. 45.

${ }^{21}$ L'historisme est pour Troeltsch la « forme de pensée spécifique de la modernité » (ibid., p. 206), audelà des Lumières.

22 Rappelons que la position de M. Weber est radicalement opposée à celle de son contemporain et ami. Elle se trouve exprimée clairement dans un texte datant de 1904 («L'objectivité de la connaissance dans les sciences et la politique sociales », dans Essais sur la théorie de la science, Paris, Plon, 1992, p. 164) : il y qualifie d'« absurdité » l'idée « que le but des sciences de la culture, aussi lointain soit-il, puisse être de former un système clos de concepts dans lequel la réalité pourrait être synthétisée selon un plan à quelque égard que ce soit définitif et dont elle pourrait ensuite être à nouveau déduite ».

23 Carl Schmitt, Glossarium, cité par Jean-François Kervégan, dans « Les ambiguïtés d'un théorème. La sécularisation de Schmitt à Löwith et retour », dans Modernité et sécularisation. Hans Blumenberg, 
L'histoire, entendue comme analyse des faits passés, consiste donc, dans cette logique, à faire apparaître ce qui, au-delà du chaos apparent, permet de dépasser la dynamique désordonnée en laquelle consiste l'historicité. Paradigme éternitiste, hypothèse du point, ces expressions analytiques sont là pour cerner et circonscrire cette entreprise qui légitime et justifie cette modalité d'analyse historique.

Les historiens participent également de cette recherche de la stabilité seule à même d'ordonner le chaos des faits. On y trouve à l'œuvre l'ambition d'une recherche de ce qui, point ou profondeur, permet d'ordonner l'histoire en la dépassant, en la stabilisant, en l'ordonnant.

Les travaux et positions d'Augustin Thierry 25 témoignent bien de cette ambition. Il convient de commencer par l'identification du « grand fait ${ }^{26}$ », la fréquentation des faits permet de construire un récit « continu ${ }^{27} »$. À partir de cette circonscription du « fait primitif», il devient possible de passer à une entreprise fondationnelle, capable de circonscrire la «base» de la nation française à partir d'un principe originel (l'hostilité, résultant de la conquête, qui structure l'histoire de France comme celle de l'Angleterre); il devient alors possible de « fixer le point précis ${ }^{28}$ » à partir duquel ordonner et donner du sens à l'histoire de France.

Je terminerai cette présentation en évoquant un texte de ce point de vue passionnant, l'important article de Fernand Braudel « Histoire et sciences sociales. La longue durée 29 ». Ce texte est particulièrement intéressant pour notre propos tant il met en scène une tension (à mon sens en partie irrésolue dans cet article) entre reconnaissance de la temporalité et recherche de la profondeur et des structures. Il faudrait, pour le commenter, en restituer le contexte sociologique relatif à la lutte d'influence entre l'histoire et cette nouvelle science qu'est l'anthropologie pour le magistère des sciences sociales. Braudel contre Lévi-Strauss, en quelque sorte, pour diriger (théoriquement, mais aussi institutionnellement) le champ des sciences sociales à l'orée des années 1960 en France. Au-delà, on pourra ne retenir ici que la prégnance du schème du stable, des profondeurs. Certes Braudel insiste sur le fait que «l'historien ne sort

Karl Löwith, Carl Schmitt, Leo Strauss, dir. Michaël Fœssel, Jean-François Kervégan, Myriam Revault d'Allonnes, Paris, CNRS Éditions, 2007, p. 107-117.

24 Ibid., p. 116.

25 Augustin Thierry (1795-1856) fait partie de cette génération d'historiens français du début du XIX siècle ayant rénové méthodologiquement les études historiques (voir Philosophie des sciences historiques. Le moment romantique, textes réunis et présentés par M. Gauchet, Paris, Seuil, 2002 [1988]). Je me référerai principalement à un article présentant l'essentiel de ces positions, « Dix ans d'études historiques », qui paraît en 1834, repris dans l'anthologie citée de M. Gauchet. Les références qui suivent faites à Thierry y renvoient.

26 Ibid., p. 47.

27 « Ma narration devint plus continue » précise-t-il, ibid., p. 51.

28 Idem.

29 Dans Annales ESC, vol. 13, n 4, oct.-déc. 1958, p. 7-37. 
jamais du temps de l'histoireso », mais il souligne aussi combien «tous les étages, tous les éclatements du temps de l'histoire [...] se comprennent à partir de cette profondeur [celle de la longue durée "presque à la limite du mouvant31"] ».

Ce paradigme de la profondeur permet aux historiens, du moment romantique à l'école des Annales, d'échapper au chaos superficiel de l'histoire étroitement politique et factuelle ; il peut aussi être vu comme symptôme d'une ontologie (aristotélicienne ?), pour laquelle l'être est repos, ordonnable, et son développement, finalisé.

Cette énumération, un peu fastidieuse peut-être, de références aura permis de circonscrire l'une des caractéristiques de la pensée héritée : sa défiance par rapport au chaos. Le chaos, loin de pouvoir définir l'être, représente un risque pour la pensée ; heureusement, il n'est que réalité superficielle, défaut de perspective. Dès lors, la stabilité et l'ordre représentent ce qu'il convient de rechercher, dans une perspective gnoséologique ou plus normative (pour Schmitt par exemple).

C'est par rapport à ce modèle qu'apparaît la singularité des positions ontologiques de Castoriadis. C'est par rapport à ce paradigme de l'ordre que ses thèses relatives à la dimension chaotique de l'être apparaissent dans toute leur radicalité : le chaos n'est plus pensé comme défaut d'ordre ou scandale moral pour devenir l'une des, sinon la caractéristique ontologique fondamentale.

\section{Strates irrégulières et complexité ou ordre et continuité}

La notion de chaos constitue donc un lieu à partir duquel saisir les présupposés de la pensée héritée, à partir duquel souligner l'emprise d'une incapacité à penser le caractère essentiellement historique de l'être au-delà de toute stabilité. Le postulat naturaliste d'un ordre et d'une stabilité, les recherches de point ou de profondeur n'apparaissent alors que comme des tentatives de dénégation face à la reconnaissance du caractère chaotique de l'être. Le champ des études historiques apparaît tout à la fois comme le lieu de cette dénégation et comme le lieu de cette reconnaissance.

On l'a annoncé, il est une autre notion, également présente dans l'œuvre de Castoriadis, dont il convient de souligner l'importance, la notion de strate. On s'y attachera en montrant comment elle introduit une nouveauté décisive, une nouvelle façon d'analyser l'historicité, rendant compte de sa dynamique interne et indépassable.

Considérer la réalité sur le mode de la stratification conduit, en effet, à reconsidérer son statut ontologique. La réalité ne peut alors pas plus être considérée comme stable que comme univoque, dont pourrait rendre compte une causalité simple, clas-

30 Ibid., p. 32.

31 Ibid., p. 18. 
sique, elle aussi univoque. La stratification appelle un nouveau modèle de causalité en même temps qu'elle permet d'échapper à l'impératif ontologique de stabilité.

Une des premières conceptualisations de cette notion de strate, en relation déjà avec des enjeux historiques, se trouve dans les textes de Georges Cuvier, entre la toute fin du XVIII ${ }^{\mathrm{e}}$ siècle et le premier tiers du XIX ${ }^{\mathrm{e}}$ siècle32. L'approche de Cuvier est scientifique, stratigraphique au sens où l'entend la géologie naissante; il est l'un des premiers à analyser le globe par l'accumulation de strates, dont le jeu et les tensions permettent de rendre compte des catastrophes auxquelles le globe fut et continue d'être soumis. L'histoire (l'histoire du globe terrestre pour Cuvier dans ses écrits géologiques) ne peut être analysée que par le biais des notions de catastrophe, de rupture, de discontinuité radicale et non par la référence à une temporalité continue. Strates, catastrophe et discontinuité s'articulent, au sein d'un système toutefois encore marqué par une ambition nomothétique régulatrice, le système de causalité antérieur reprenant ses droits après l'épisode catastrophique. Il y a cependant chez Cuvier une attention aux révolutions géologiques, aux catastrophes qui rythment fondamentalement l'histoire essentiellement discontinue de la Terre. Le temps historique ne peut être conçu comme continuum, il convient de penser l'articulation entre stabilité et discontinuité sans qu'aucune résolution définitive ne soit envisageable. Le modèle aristotélicien pour lequel le repos est la fin du mouvement ne peut rendre compte de la temporalité historique.

L'enjeu discontinuiste de la notion de strate se retrouve chez Nietzsche, dernière référence faite ici pour cerner le sens de l'usage et de la référence à cette notion. On retrouve cette notion à de très nombreuses reprises dans les textes de ce que l'on peut cerner comme le deuxième moment nietzschéen, entre l'influence wagnérienne et les derniers textes de la fin des années 1880 .

Dans Humain, trop humain en particulier, au §223, un passage illustre cette nouvelle façon de concevoir l'histoire comme résultat d'une stratification complexe. Le texte commence par l'affirmation selon laquelle "[1]'observation directe de soimême ne suffit pas pour se connaître 33 »; saisir le « flot de notre être apparemment le plus singulier 34 » conduit à la nécessaire prise en compte de l'histoire. Il convient " pour comprendre l'histoire [de] rechercher les vestiges vivants des époques historiques35 ». S'exprime ici une caractéristique essentielle du modèle de la strate, qui consiste à cerner l'actualité de chacune d'entre elles, au-delà de leur caractère enfoui, mais sans recherche d'exclusivité et de réduction. Car les strates jouent sans cesse au pluriel. La suite du texte souligne combien les strates «continuent» d'agir de

\footnotetext{
32 Dans le Discours sur les révolutions de la surface du globe, et sur les changements qu'elles ont produits dans le règne animal, Paris, Christian Bourgois, 1985 [1825].

33 Friedrich Nietzsche, Humain, trop humain, Paris, Gallimard, 1968, p. 113.

34 Idem.

35 Idem.
} 
concert, sans qu'il soit possible d'en ordonner, et encore moins d'en hiérarchiser l'influence. De telle sorte qu'il sera possible de « redécouvrir les aventures vagabondes de cet ego en devenir et métamorphose $36 »$.

La mobilisation théorique du modèle de la strate conduit donc à penser l'historicité complexe, de l'ego chez Nietzsche, de la Terre chez Cuvier. Mais il faut souligner qu'il ne s'agit nullement d'une historicité pensée comme moment transitoire, comme étape d'une analyse finalisée par la recherche d'un niveau architectonique. En effet, la stratification de la réalité conduit à reconnaître l'inexistence d'une quelconque infrastructure. Nous nous trouvons face à une multiplicité de strates jouant indéfiniment les unes sur les autres, dans une rémanence de leurs effets.

Il convient maintenant, deuxième moment plus heuristique annoncé, de préciser les attendus historiques de la logique conceptuelle qui vient d'être rappelée. Cela va nous conduire à dépasser le cadre de l'analyse de la pensée héritée en auscultant l'historicité indépassable de la réalité historique, à repérer comment son organisation stratifiée rend compte du caractère dynamique et toujours à être de la réalité socialhistorique.

Précisons qu'il ne saurait être question de repérer des traces explicites d'influence (à l'exception éventuelle du cas d'E. P. Thompson); les repérages qui suivent relèvent bien plus de l'usage sauvage que permet une œuvre, usage qui ne se justifie que par les perspectives qu'il rend possibles.

\section{Strate et rémanence}

La première référence, Carlo Ginzburg, sera l'occasion de retrouver la tension évoquée précédemment entre reconnaissance de l'historicité des sociétés humaines et tentation-tentative d'identification de lieux pérennes structurant l'organisation de ces mêmes sociétés humaines.

Ginzburg s'est d'abord fait connaître par ses travaux relevant de la micro-histoire, avec Le fromage et les vers 37 en particulier. Il a par la suite tenté de préciser son approche méthodologique dans «Signes, traces, pistes 38 ». Si la notion de trace y a des enjeux essentiellement méthodologiques, on la retrouve, associée alors à la notion de strate, dans un livre qui va nous intéresser, Le sabbat des sorcières ${ }^{39}$, qui prolonge et

\footnotetext{
36 Idem.

37 Carlo Ginzburg, Le fromage et les vers. L'univers d'un meunier frioulan du XVI siècle, Paris, Aubier, 1980 [1976].

38 Carlo Ginzburg, «Signes, traces, pistes. Racines d'un paradigme de l'indice », dans Le Débat, vol. 6, $\mathrm{n}^{\circ} 6,1980$, p. 3-44.

39 Carlo Ginzburg, Le sabbat des sorcières, Paris, Gallimard, 1992.
} 
Réalité, strates et chaos : enjeux historiographiques de l'œuvre de Castoriadis | Nicolas PIQUÉ

transforme radicalement des recherches initiées dans Les batailles nocturnes. Sorcellerie et rituels agraires en Frioul, $X V I^{e}-X V I I^{e}$ siècles ${ }^{40}$.

L'objet et son exposition sont, comme souvent chez Ginzburg, complexes et difficiles à résumer ; je vais pourtant m'y risquer. Il s'agit de rendre compte d'une anomalie surgie au sein de l'analyse de la sorcellerie à laquelle Ginzburg s'était attachée dans un premier temps dans Les batailles nocturnes. Certains récits recueillis par l'Inquisition lui apparurent trop singuliers pour n'être pas les traces d'influences lointaines de pratiques chamaniques; ces récits racontent des batailles nocturnes, sorties extatiques des corps dont l'enjeu concerne la fertilité des récoltes. L'entreprise du Sabbat des sorcières prend alors des proportions et un style méthodologique tout à fait singuliers pour réussir à circonscrire, puis analyser les voies par lesquelles se transmettent et se construisent ces récits de bataille nocturnes. Il s'agira de circonscrire la "couche mythique et rituelle très profonde d'où naquirent les croyances populaires qui ont été par la suite intégrées de force dans le sabbat ${ }^{41} »$.

Il est hors de question ici de détailler cette «imbrication de morphologie et d'histoire 42 » cherchant à articuler " événement » et « lente sédimentation 43 ». Ginzburg commence par pointer la résurgence, jusqu'au XVII ${ }^{\mathrm{e}}$ siècle, de procès de sorcellerie révélant de profondes similitudes; l'étalement dans le temps et dans l'espace de ces récits (dans lesquels les accusés racontent s'être rendus au sabbat, en présence du diable et en se livrant à des festins, orgies et autres profanations) constitue une énigme dont le livre va constituer la tentative de résolution. Au-delà des enjeux méthodologiques et épistémologiques aussi intéressants que parfois déroutants, ne seront ici retenus que deux aspects, entrant en tension de façon finalement peut-être problématique. D'abord, donc, le vocabulaire, qui emprunte à la géologie : il est question de couche, de sédimentation, d'affleurement, de strate. Le vocabulaire de la strate est omniprésent. Il permet de rendre compte de la coexistence, de la superposition de différents schèmes culturels, Ginzburg s'attachant plus particulièrement à retracer l'avènement du plus archaïque, à partir d'une lointaine origine sibérienne. Dans un premier temps, son entreprise semble alors s'apparenter à celle qui fut la sienne dans Le fromage et les vers, et qui rappelle celle d'E. P. Thompson à laquelle il sera fait référence plus bas : contre la « violence historique44 » d'imposition de représentations sociales à la fois dominantes, normatives et uniformisantes, l'historien a vocation à rappeler la pluralité et la complexité des strates composant les représentations sociales. La réalité sociale apparaît alors comme le résultat de cette stratification historique et irrégulière parce que résultat aussi des rapports de force sociaux au tra-

40 Carlo Ginzburg, Les batailles nocturnes. Sorcellerie et rituels agraires en Frioul, XVI ${ }^{e}$-XVII siècles, Lagrasse, Verdier, 1980.

${ }^{41}$ Carlo Ginzburg, Le sabbat des sorcières, op. cit., p. 22.

42 Ibid., p. 267.

43 Ibid., p. 269.

44 Ibid., p. 24. 
vers desquels elle se constitue. La réalité historique résulte de cette superposition non systématisable, de la rémanence aussi de ses composantes.

Dans «Complexité, magmas, histoire45», Castoriadis ébauche un modèle d'analyse analogue : après avoir dégagé les attendus ontologiques et épistémologiques (y compris dans le champ mathématique) de "l'hétérogénéité des axiomes 46 », il en circonscrit et caractérise les termes et attendus historiques. En prenant le cas de l'histoire moderne européenne, il identifie quatre «principes d'origine historique diverse et essentiellement hétérogènes 47 ", qui s'insèrent de surcroît dans un monde à la fois romanisé et christianisé. Cette hétérogénéité est décisive, le jeu indéfini de leurs relations rendant compte du caractère toujours à être de l'être.

Mais il convient de reconnaître, dans l'entreprise même de Ginzburg, un autre aspect qui va nous ramener à l'un des enjeux déjà évoqués de la pensée héritée, la recherche du stable et de l'anhistorique. L'aveu peut étonner, mais Ginzburg reconnaît: «il y a bien longtemps je m'étais proposé de démontrer de manière expérimentale, d'un point de vue historique, l'inexistence de la nature humaine ; je me suis trouvé, vingt-cinq ans plus tard, soutenir une thèse exactement inverse 48 ». L'analyse des strates, dont on a souligné les enjeux chaotiques, au sens où Castoriadis souligne la dimension chaotique de l'être, peut également mener à l'entreprise diamétralement opposée d'une recherche de strate fondamentale, originaire et finalement pérenne et naturelle.

Cette hypothèse d'une strate naturelle illustre l'inertie de la pensée héritée dans le champ historique. Alors même qu'une partie des analyses de Ginzburg conduit à penser la réalité historique comme intrinsèquement instable en raison de la multiplicité instable des strates la composant, la quête et l'espoir d'une stabilité presque substantielle et pérenne viennent limiter cette première reconnaissance.

Une telle supposition n'obère toutefois pas ce que les travaux de Ginzburg ont permis de faire apparaître, soit une représentation complexe de la réalité historique, représentation qui empêche de la considérer comme système dialectisable de systèmes. La multiplicité de ses composantes, leur caractère rémanent, les rapports de normalisation toujours incomplets qui les transforment, tout cela concourt à penser le caractère dense de la réalité historique. La stratification irrégulière conduit à une pensée du caractère essentiellement historique ou dynamique de la réalité, à l'opposé d'une représentation stable ou substantielle.

45 Cornelius Castoriadis, « Complexité, magmas, histoire. L'exemple de la ville médiévale », dans Les carrefours du labyrinthe, V. Fait et à faire, Paris, Seuil, 1997.

46 Ibid., p. 215.

47 Ibid., p. 217 (je souligne).

48 Carlo Ginzburg, Le sabbat des sorcières, op. cit., p. 36. 


\section{Émergence et cercle de la création}

La question du statut de la réalité historique constitue encore l'enjeu de la seconde référence historique, les travaux d'E. P. Thompson ; moins en tension par rapport aux positions de Castoriadis, elle va nous permettre d'aborder la question, sensible aux dires même de Castoriadis, du cercle de la création.

Il faut peut-être rappeler que Thompson publia dans le $\mathrm{n}^{\circ} 5$ de Libre un article important, intitulé «Temps, discipline du travail et capitalisme industriel », lien explicite entre les deux penseurs. Ce n'est toutefois pas à ce texte que je vais me référer, mais à son maître livre qu'est La formation de la classe ouvrière anglaise 49 . Le titre l'indique assez, Thompson y analyse un lent processus d'émergence; pour ce faire, il se lance dans une vaste et dense analyse des influences et rémanences des diverses traditions qui vont permettre d'expliquer la façon dont se transforme le champ social en Angleterre entre les XVIII ${ }^{\mathrm{e}}$ et XIX ${ }^{\mathrm{e}}$ siècles. L'insistance sur le processus est rappelée dès la préface : "Le titre de ce livre est maladroit, mais il a le mérite d'être adéquat. Le mot "formation" [making] indique que l'objet de cette étude est un processus actif, mis en œuvre par des agents tout autant que par des conditions $5^{\circ} \gg$; il convient de mesurer la part que prennent à sa dynamique les traditions dont Thompson va étudier à la fois la teneur et la force dynamique.

De ce point de vue, son livre est une plongée assez passionnante, parce que très informée et donc très proche, dans les évolutions concrètes des acteurs de la transformation, de l'émergence de la classe ouvrière. Il suit, comme un enquêteur, la rémanence, la postérité, la continuité de diverses «traditions » (c'est le terme qu'il utilise), comme les niveleurs, les millénaristes, les jacobins, etc. Certaines disparaissent pendant quelques années ou décennies, pour réapparaitre à l'occasion d'un nouveau contexte, prenant la place d'une autre dont l'influence décroît pour quelque temps. La virtuosité de l'analyse et son information plongent le lecteur au centre de cette réalité mouvante dont on finit presque par sentir le flux et les dynamiques.

Le problème qu'aborde alors Thompson, et qui va nous ramener à la question du cercle de la création, s'énonce de la sorte : il s'agit «d'aborder la complexité et la continuité de ces traditions » pour cerner les conditions d'émergence du nouveau : « il nous faut essayer de comprendre deux choses : la continuité des traditions et la modification de leur contexte $51 »$. L'attachement à cette relation dialectique (au sens de relation non univoque) structure nombre d'analyses du livre. Lequel met en œuvre un schème singulier d'étude du nouveau, qui se trouve pensé sur le mode de la combinaison, non pas aléatoire, mais non prédictible, des traditions dont les chapitres suivent les flux et les reflux.

\footnotetext{
49 Edward Palmer Thompson, La formation de la classe ouvrière anglaise, Paris, Seuil, 1988 [1963].

50 Ibid., p. 15 ; est ici également circonscrit l'enjeu d'une analyse de l'agency/agentivité.

51 Ibid., p. 34.
} 
Le nouveau et la transformation de la structuration du champ social résultent ainsi de ce qu'il est possible d'appeler une logique des effets. Jamais Thompson ne cherche à surdéterminer les destins ou les destinées des traditions ; il en suit les histoires qui interagissent les unes avec les autres pour créer de nouvelles configurations inédites et inattendues. La formation de la classe ouvrière apparaît alors comme le résultat non prédictible de causalités plurielles et hétérogènes. La logique du processus que décrit Thompson échappe à toute logique causale classique; les formules par lesquelles Castoriadis présente, dans «La source hongroise » en particulier, sa critique de la causalité classique, en insistant sur "l'excès de l'"effet" sur les "causes52" ", paraissent tout à fait proches de ce que Thompson circonscrit. Il y a de la nouveauté parce qu'il y a plus dans les effets produits par la rencontre des traditions, que dans ce que les traditions comportaient chacune.

La lecture rapide de ce texte nous reconduit donc au schème de l' " excès » (formulation par ailleurs peut-être plus efficace et heuristique que celle qu'il emploiera plus tard, présentant la création ex nihilo, en la distinguant des créations in nihilo et cum nihilo53). Thompson, en insistant sur le lien complexe et finalement assez obscur entre tradition et nouveauté, peut s'inscrire dans ce débat, en le travaillant "from below », pour reprendre une formule par laquelle il caractérisa méthodologiquement (et politiquement) son travail.

L'énigme de la création de formes nouvelles renvoie au statut de la réalité. Cette dernière est constituée de composantes dont les temporalités sont différentes, irréductibles les unes aux autres. La complexité de la réalité historique, qui tient aux relations de ses composantes, explique l'impossibilité de réduire l'émergence du nouveau à un schème causal simple.

\section{Réalité stratifiée ou société chaotique?}

La dernière série de références, faites au paradigme de l'histoire connectée à travers deux de ses représentants que sont Sanjay Subrahmanyam et Serge Gruzinski, dessine un lien incontestablement plus lâche à l'égard des positions de Castoriadis. L'enjeu de reconnaissance de l'historicité y est cependant bien présent, en lien encore avec une conception de la réalité historique décrite alors comme métisse, et cette reconnaissance est fondamentale. Une divergence apparaîtra toutefois dans l'usage de la notion de chaos (pourtant lien a priori entre les corpus), dont l'enjeu concerne alors plus le statut de l'ordre social que celui de la réalité historique.

$5^{2}$ Cornelius Castoriadis, «La source hongroise », dans Le contenu du socialisme, Paris, Union Générale d'Éditions, 1979, p. 383.

53 Voir « Complexité, magmas, histoire... », dans Les carrefours du labyrinthe, $V$, op. cit., p. 212, l'une des occurrences de cette problématisation. 
Subrahmanyam est spécialiste de l'étude des relations entre l'Inde et l'Europe aux $\mathrm{XVI}^{\mathrm{e}}$ et $\mathrm{XVII}^{\mathrm{e}}$ siècles. Il est également le promoteur d'un programme de travail qu'il présente sous le nom d' « histoire connectée 54 ». La question qui constitue l'objet de cet article-manifeste est la notion d'incommensurabilité. Ce faisant, il s'inscrit dans le cadre de la critique des modèles historiographiques traditionnels, accusés de dépendre d'un centrement européen abusif. Ces critiques ont, pour certaines, conduit à souligner l'indépendance des cultures, aboutissant à une supposée incommensurabilité. C'est à cette thèse que s'en prend Subrahmanyam, sans pour autant, on s'en doute, réactiver une position ou une logique européocentrée. Il présente alors ses positions comme résultat d'un double refus, celui d'une prééminence européenne, mais celui aussi d'un relativisme postmoderne (qu'il identifie parfois à certaines productions des Subaltern studies).

La position du problème est la suivante. Après avoir identifié les références lui semblant permettre de penser le repli sur soi des cultures (Herder, entre autres), après avoir rappelé les travaux de N. Wachtel sur le processus d'acculturation, après avoir donc souligné les interactions composant le tissu de l'histoire du monde, il en vient à préciser sa position :

Ce que je veux dire, au fond, est assez simple. On a pu le comprendre à travers mes commentaires sur l'approche de Bernard Cohn. La plupart des théorisations de «l'incommensurabilité culturelle » reposent sur une conception structuraliste de la culture et se heurtent de ce fait à une question qui est centrale pour les historiens : celle du changement, de l'évolution55.

Cette critique du structuralisme, du déni de l'histoire qu'induit ce modèle, a pour enjeu la reconnaissance d'une historicité indépassable du social-historique. Une telle critique, révélant le même enjeu, constitue l'un des moments importants du début de L'institution imaginaire de la société. Castoriadis y souligne combien le structuralisme est fondamentalement incapable de rendre compte et de penser la réalité irréductible de l'événement. Or le travail de l'histoire consiste précisément, pour Castoriadis comme pour Subrahmanyam, à circonscrire et penser cette solution de continuité, laquelle définit l'objet même de la discipline historique.

Plusieurs de ses travaux s'inscrivent dans une dimension d'abord synchronique, cherchant à décrypter, par exemple, les conditions des rencontres impériales entre puissances européennes et asiatiques ${ }^{5}$. Même si elles n'emploient pas explicitement

54 Voir l'une des présentations dans l'article-manifeste «Par-delà l'incommensurabilité : pour une histoire connectée des empires aux temps modernes », Revue d'histoire moderne et contemporaine, vol. $5, \mathrm{n}^{\mathrm{o}}$ 54-4bis, 2007, p. 34-53.

55 Ibid., p. 48.

56 Voir par exemple Sanjay Subrahmanyam, «Du Tage au Gange au XVI ${ }^{\mathrm{e}}$ siècle : une conjoncture millénariste à l'échelle eurasiatique », Annales. Histoire, Sciences Sociales, vol. 56, nº 1, 2001, p. 51-84. 
la notion de strate, les analyses de Subrahmanyam s'attachent à souligner combien les mondes asiatiques plongent eux aussi leurs racines dans une épaisseur temporelle, bien souvent déniée par les puissances européennes ${ }^{57}$, faisant intervenir une épaisseur stratifiée. Le programme de l'histoire connectée permet à Subrahmanyam de souligner combien les sociétés ne constituent jamais des totalités closes sur elles-mêmes et consistantes. Parce qu'elles sont des sociétés historiques, mais aussi parce qu'elles sont toujours en lien, en relation avec d'autres, qui agissent sur elles, qui connaissent un effet en retour dont on ne peut à l'avance mesurer l'enjeu.

Les travaux de Serge Gruzinski s'inscrivent en partie dans le même contexte théorique et épistémologique. Il est spécialiste de l'histoire du Mexique et de la monarchie espagnole dans sa configuration impériale aux $\mathrm{XVI}^{\mathrm{e}}$ et XVII ${ }^{\mathrm{e}}$ siècles (15801640). Il a également dernièrement mené une réflexion plus générale et en partie décontextualisée, menant à la rédaction de La pensée métisse ${ }^{8}$.

Il définit le centre de son travail par l'étude des «mondes mêlés de la monarchie catholique 59 »; il souligne le « réagencement de l'histoire » que l'on peut y trouver, contribuant à une « pluralité des histoires ${ }^{60}$ ». Le travail de l'historien est comparable à celui de l'électricien chargé de (re)connecter ce qui l'a été pour être ensuite séparé et oublié. L'analyse de la monarchie espagnole, dans la complexité et l'interaction des liens qui s'y tissent entre trois continents, lui apparaît comme un champ d'étude tout à fait remarquable de ce point de vue, celui d'une compression des distances, d'une planétarisation aboutissant à des interactions, des effets en retour multiples et incessants, ainsi qu'à de constants réagencements. Il s'agit d'en « parcourir [1]e tissu dans toutes ses épaisseurs ${ }^{61} »$.

Dans La pensée métisse, il précise encore ce programme de recherche. Les concepts les plus opératoires sont ceux de «chaos », de «complexité » et de « désordre », de « bifurcation », le modèle théorique n'étant par ailleurs pas totale-

57 «Ce monde plongeait ses racines dans des "profondeurs temporelles” et des étendues spatiales spécifiques, qui ne coïncidaient que marginalement avec les juridictions coloniales. Ce sont précisément ces bassins d'historicité, débordant largement le cadre anachronique des histoires nationales, que l'histoire connectée permet de mettre au jour. L'histoire connectée nous rappelle en effet, si besoin en était, que la forme impériale d'État n'était nullement l'apanage de l'Europe. L'Asie du Sud-Est était d'évidence, à l'aube de l'arrivée des Portugais dans l'Océan Indien, un monde profondément impérial, travaillé depuis des siècles par des processus de centralisation politique (fiscale, militaire et idéologique)»: Romain Bertrand commente ainsi les travaux de Subrahmanyam («Rencontres impériales. L’histoire connectée et les relations euro-asiatiques », Revue d'histoire moderne et contemporaine, "Histoires globales », vol. $5, \mathrm{n}^{\circ}$ 54-4bis, 2007, p. 82).

58 Serge Gruzinski, La pensée métisse, Paris, Fayard, 1999.

59 Serge Gruzinski, "Les mondes mêlés de la Monarchie catholique et autres "connected histories" ", dans Annales. Histoire, Sciences Sociales, $56^{\mathrm{e}}$ année, $\mathrm{n}^{\mathrm{o}}$ 1, 2001, p. 85-117.

60 Idem.

61 Ibid., p. 116. 
ment systématisé. Il s'agit de penser les « formes de temporalité et d'historicité62 » résultant de «l'empilement des époques ${ }^{63} »$.

Pour prendre un exemple des analyses de Gruzinski, citons la Vierge de Guadalupe, dont il analyse minutieusement ${ }^{64}$ les diverses composantes, les différentes strates, qui doivent à la Conquête comme aux représentations autochtones, dans une unité aussi nouvelle qu'irréductible à ses composantes.

Le modèle du «mélange », de l'« empilement des époques » et des « différentes temporalités 65 », créant ce que Gruzinski caractérise comme «société fractale 66 », renvoie au schème de la stratification que l'on a déjà relevé. Cette stratification fait intervenir des éléments hétérogènes, comme le souligne l'analyse de la Vierge de Guadalupe. Ce que l'on a circonscrit comme stratification irrégulière, Gruzinski le désigne comme réalité métisse pour en faire une caractéristique fondamentale des sociétés humaines.

On retrouve alors la conception singulière de la réalité historique mise à jour chez Castoriadis, une réalité mêlée, complexité chaotique67, dirait Gruzinski, faite d'influences, de conquêtes, d'empilements et de rémanences, d'interactions et d'effets en retour. Les ensembles produits sont non consistants, complexes ou métis, au sens où aucun de leurs éléments ne peut prétendre au rang architectonique d'infrastructure pérenne.

L'histoire connectée ne vise aucune forclusion de l'histoire. Ce point est, pour notre propos, décisif.

Il s'agit là d'un des intérêts de l'histoire connectée que de lancer un programme de recherche ne visant pas à établir de stabilité finale, mais cherchant au contraire à mettre en évidence les dynamiques complexes, les émergences inattendues de nouvelles significations. À l'instar du vers d'un personnage d'un roman de Mario de

\footnotetext{
62 Serge Gruzinski, La pensée métisse, op. cit., p. 50.

63 Ibid., p. 44.

64 Dans La colonisation de l'imaginaire. Sociétés indigènes et occidentalisation dans le Mexique espagnol, XVI $-X V I I I^{e}$ siècle, Paris, Gallimard, 1988 ; voir, du même, La guerre des images de Christophe Colomb à Blade Runner (1492-2019), Paris, Fayard, 1990 ainsi que « Colonisation et guerre des images dans le Mexique colonial $\left(\mathrm{XVI}^{\mathrm{e}}-\mathrm{XVII}{ }^{\mathrm{e}}\right.$ siècle)», dans Transferts culturels et métissages. Amérique/Europe $X V I^{e}-X X^{e}$ siècle, dir. Laurier Turgeon, Denis Delâge, Réal Ouellet, Québec, Presses de l’Université Laval, 1996.

65 Serge Gruzinski, La pensée métisse, op. cit., p. 33, 44, 67.

66 Serge Gruzinski, "Les Indiens du Mexique face à la conquête espagnole : du chaos aux premiers métissages ", dans Destins croisés. Cinq siècles de rencontres avec les Amérindiens, dir. Joëlle Rostkowski, Sylvie Devers, Paris, Unesco-Albin Michel, 1992, p. 57 ; Gruzinski utilise aussi la notion de «société baroque» («Événement, bifurcation, accident, hasard... Regards sur l'histoire depuis les périphéries de l'Occident», dans Relier les connaissances. Journées thématiques conçues et animées par Edgar Morin, Paris, Seuil, 1999, p. 313).

67 On reviendra plus bas sur le sens de cette expression.
} 
Andrade, « Je suis un Tupi qui joue du luth 68 », les mondes dans lesquels nous vivons résultent de cette complexité tout à la fois chaotique et organisée. Dès lors, le travail de l'historien est à la fois diachronique (analyser le choc de la Conquête, par exemple, pour analyser la façon dont émerge un monde nouveau, au sens arendtien) et synchronique (identifier, par exemple, les strates composant le culte de la Vierge de Guadalupe). L'histoire ne peut alors qu'être connectée, reconstituant les lignes d'échange, circonscrivant les traces qui en résultent dans chaque partie du monde, monde qu'il faut alors comprendre comme tissu de relations incessantes aux effets imprévisibles.

Relèvent aussi de cette même configuration les recherches de Romain Bertrand ${ }^{69}$ et de Denys Lombard70, pour ne prendre que deux références travaillant la même aire culturelle, l'Asie du Sud-Est. Lombard en particulier souligne combien la compréhension de l'Indonésie se doit de délaisser l'ordre chronologique classique: "Cherchant à privilégier la notion de strate qui, dans le cas présent au moins, nous paraît éclairante, nous avons présenté les différentes nébuleuses mentales dans l'ordre même où elles affleurent71. ") L'analyse de ces strates (culture indienne, influences chinoise et islamique, civilisation européenne enfin) et de leur enchevêtrement permet seule de comprendre la dynamique intrinsèque et mélangée de ce « monde des réseaux ${ }^{72} »$.

Pour autant, l'emploi, par Gruzinski en particulier, de la notion de chaos reste problématique, eu égard en tout cas à son usage castoriadien. La notion de chaos revient en effet à de très nombreuses reprises dans La pensée métisse; elle permet à Gruzinski de rendre compte de la complexité de la réalité, résultat de mélanges et de syncrétismes non univoques. L'objet de l'historien consiste alors à étudier des «situations instables, imprévues et imprévisibles 73 ». On pourrait donc discuter de l'emploi par Gruzinski de la notion de chaos qui désigne le mode d'être d'une société analysée comme tout chaotique, ce qui n'est pas le cas chez Castoriadis. Ce dernier,

68 Cité dans La pensée métisse, op. cit., p. 17.

69 En témoigne l'intérêt pour l'analyse des moments de rencontre entre puissances coloniales et peuples conquis. Ces moments ne peuvent être réellement compris, "à parts égales" (Romain Bertrand, L'Histoire à parts égales. Récits d'une rencontre Orient-Occident (XVI -XVII siècle), Paris, Seuil, 2011) qu'en prenant en compte les strates spécifiques de l'histoire des pays conquis, pour éviter de ne voir dans ces pays que des terres vierges à l'historicité vide à l'instant de la rencontre : voir Romain Bertrand, " "Des gens inconvenants". Javanais et Néerlandais à l'aube de la rencontre impériale » (Actes de la recherche en sciences sociales, vol. 1, n¹71-172, 2008, p. 104-121), "Rencontres impériales. L'histoire connectée et les relations euro-asiatiques », (op. cit., p. 69-89), "La rencontre coloniale, une affaire de mœurs ? L'aristocratie de Java face au pouvoir hollandais à la fin du XIX ${ }^{\mathrm{e}}$ siècle » (Genèses, vol. $2, \mathrm{n}^{\circ} 43$, juin 2001 , p. 32-52).

70 Denys Lombard, Le carrefour javanais. Essai d'histoire globale, Paris, Éditions de l'EHESS, 1990, 3 vol.

71 « Avant-propos », dans ibid., vol. 1, Les limites de l'occidentalisation, p. 9.

72 Ibid., vol. 3, L'héritage des royaumes concentriques, p. 155.

73 Serge Gruzinski, La pensée métisse, op. cit., p. 104. 
on l'a évoqué, souligne combien la société instituée possède un ordre, et finalement une consistance, fût-elle régionale et limitée. Il y a donc divergence quant à la modalité et au degré d'institutionnalité, en quelque sorte. En soulignant la dimension métisse, bigarrée de la réalité sociale, l'analyse de Gruzinski est redevable d'un autre modèle théorique que celui de Castoriadis, un modèle pour lequel les notions de réseau, de flux et d'échange paraissent fondamentales. Au prix, du point de vue des analyses castoriadiennes, de l'importance et de l'enjeu de la notion de clôture. À l'inverse, l'intérêt de Subrahmanyam et de Gruzinski pour les phénomènes d'échange et de métissage comme vecteur d'institution de sens peut permettre de circonscrire, en retour, un défaut dans les analyses de Castoriadis, en partie aveugle à cette dimension dans l'élaboration de ce qui permet à une société de se singulariser. On peut en effet noter que les analyses de ce dernier restent souvent focalisées sur les conditions internes de la nouveauté, dont l'insuffisance le conduit d'ailleurs à faire l'hypothèse de créations ex nihilo. Ce faisant, ses analyses ne cherchent peut-être pas assez à rendre compte de l'historicité des sociétés par les phénomènes d'emprunt et de rencontre, auxquels s'attachent précisément aussi bien Subrahmanyam que Gruzinski.

Travailler les schèmes castoriadiens dans ce sens permettrait alors d'échapper aux difficultés posées par la notion de création ex nihilo. L'une d'entre elles, et pas la moindre, me paraît résider dans la difficulté d'en repérer une occurrence historique. Alors qu'il s'intéresse précisément à l'analyse d'une rupture majeure dans l'histoire occidentale, la Renaissance, Hans Blumenberg74 relève pourtant que "l'idée d'un commencement absolu [...] est aussi peu rationnelle que n'importe quelle creatio ex nihilo75 ». Ce jugement est d'autant plus intéressant qu'il est porté dans le cadre d'une critique du théorème de sécularisation, soutenu par C. Schmitt dans sa Théologie politique ${ }^{6}$, lequel tente à l'inverse de délégitimer toute nouveauté à la Modernité en réduisant ses principes, supposés novateurs, à de simples reformulations de thèses. Le sens des analyses de Blumenberg consiste donc bien à proposer un schème d'analyse de la nouveauté, qui la légitime tout en évitant en même temps ce qu'il considère comme les affres du modèle de la creatio ex nihilo. Son intérêt pour les seuils historiques, pendant lesquels se jouent les bouleversements, est à la mesure de la complexité consistant, dans le détail foisonnant de ses analyses, à essayer de pointer les transformations sourdes et complexes menant d'une époque à une autre. Mais « il n'existe pas de témoins d'un bouleversement d'époque. Un tournant d'époque est une limite imperceptible qui n'est liée à aucune date ou événement marquant 77 ». Et ce d'autant que «l'objectivation historique » (les progrès de l'érudition, dont

\footnotetext{
74 Hans Blumenberg, La légitimité des Temps modernes, Paris, Gallimard, 1999.

75 Ibid., p. 157.

76 Jean-François Kervégan en propose une lecture dans « Les ambiguïtés d'un théorème. La sécularisation de Schmitt à Löwith et retour », op. cit.

77 Hans Blumenberg, La légitimité des Temps modernes, op. cit., p. 533.
} 
Blumenberg est un représentant éminent !) semble induire le "nivellement théorique $^{78} \gg$ des notions d'époque et de seuil historique.

L'analyse des modèles théoriques à même de rendre compte de la nouveauté semble dès lors devoir choisir entre la critique schmittienne, la radicalité des propositions de Castoriadis, en distinguant peut-être les deux formulations relevées plus haut, le nivellement érudit de Blumenberg et enfin l'attention aux rencontres de l'histoire connectée.

Nul doute que l'on se trouve là face à une alternative théorique importante quant aux statuts et même au mode d'être de la réalité sociale. On se contentera ici de retenir des analyses de Gruzinski leurs attendus historiques entérinant l'historicité indépassable, pour laquelle l'analyse du temps historique est détachée de tout paradigme d'ordre et de stabilité pérenne.

Au-delà de l'ébauche du débat par laquelle on vient de terminer, le principal enjeu historique, ou historiographique, des positions de Castoriadis concerne le statut de la réalité. Sa constitution irrégulière, résultat d'une succession d'institutions, explique l'historicité essentielle du social-historique; on peut également rendre compte de cette dernière par le caractère chaotique de l'être. La systématicité de ces thèses, liant propositions ontologiques et attendus historiographiques ou épistémologiques, circonscrit la singularité et l'intérêt de l'œuvre de Castoriadis, ouvrant un possible dépassement du seul niveau descriptif ou analytique des études historiques. On pourrait rajouter des attendus politiques, plus obsédants encore à l'heure du consensus libéral pour lequel « There is no alternative».

Pour autant, ce dépassement ne présuppose nullement la possibilité d'un ensemble totalement et définitivement consistant et dense de positions disant la vérité ultime de la réalité. Il ne résulte pas plus, comme chez Marx, d'une logique de l'histoire à laquelle on ne saurait échapper en dernière instance. Se dessine alors un programme de recherche alliant descriptions régionales ou épocales et reconnaissance du caractère abyssal ou insondable en dernière instance de la réalité social-historique. Ce sansfond n'obère en rien l'enquête, il en dessine les limites, l'analyse de ce qui fut n'empêchant en rien d'imaginer ce qui pourrait être.

78 Ibid., p. 534. On peut ici également citer Alexandre Koyré, lui aussi sensible à la complexité des positions de ceux que l'on présente de manière univoque comme des novateurs; voir en particulier «L'apport scientifique de la Renaissance » et «Galilée et la révolution scientifique du XVII ${ }^{\mathrm{e}}$ siècle », tous deux repris dans Études d'histoire de la pensée scientifique, Paris, Gallimard, 1973. 